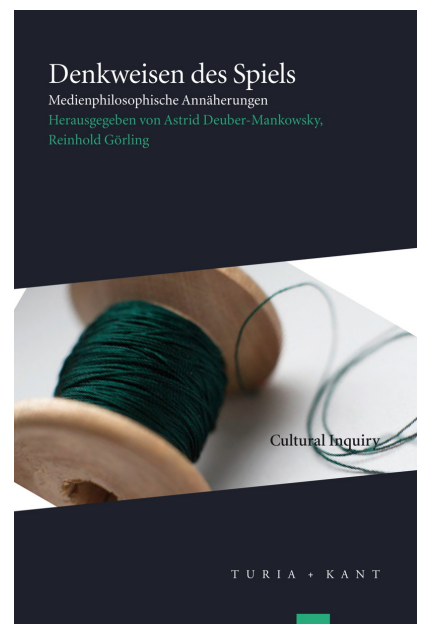

Denkweisen des Spiels: Medienphilosophische Annäherungen, hg. v. Astrid Deuber-Mankowsky und Reinhold Görling, Cultural Inquiry, 10 (Wien: Turia + Kant, 2017), S. 213-37

\section{ASTRID DEUBER-MANKOWSKY}

\section{Variationen des Spiels}

\section{Seeing Red von Su Friedrich mit Deleuze, Guattari und Benjamin}

\author{
ZITIERVORGABE:
}

Astrid Deuber-Mankowsky, »Variationen des Spiels: Seeing Red von Su Friedrich mit Deleuze, Guattari und Benjamin $\ll$, in Denkweisen des Spiels: Medienphilosophische Annäherungen, hg. v. Astrid DeuberMankowsky und Reinhold Görling, Cultural Inquiry, 10 (Wien: Turia + Kant, 2017), S. 213-37 <https://doi.org/10.37050/ci-10_12>

ANGABE ZU DEN RECHTEN:

(C) by the author(s)

This version is licensed under a Creative Commons AttributionShareAlike 4.0 International License. 


\title{
VARIATIONEN DES SPIELS
}

\author{
Seeing Red von Su Friedrich mit Deleuze, \\ Guattari und Benjamin \\ Astrid Deuber-Mankowsky ${ }^{1}$
}

In seinem posthum veröffentlichten Videointerview Abécédaire antwortet Gilles Deleuze Claire Parnet an einer Stelle: »Sollte man mich nämlich fragen: /Welchen philosophischen Begriff hast du produziert, wenn du schon davon sprichst, Begriffe zu erschaffen? Zumindest einen sehr wichtigen haben wir produziert, den des $>$ Ritornells $\triangleleft{ }^{2}{ }^{2}$ Ein Ritornell ist zunächst eine kleine Melodie, ein Refrain, ein »Tralala «, das, wie Deleuze ausführt, bei drei Gelegenheiten gesummt wird: Wenn man zuhause ist und sein Revier abgeht, wenn man versucht in sein Zuhause zurückzukehren, beim Einbruch der Nacht, der »Stunde der Angst «. ${ }^{3}$ Und drittens singt man es, wenn man von Zuhause fortgeht und sein Herz mitnimmt. Das Ritornell hat, wie daraus deutlich wird, mit dem Problem des Territoriums zu tun, mit dem Eintritt und dem Austritt aus einem Territorium und mit dem Problem der Deterritorialisierung. Deleuze und Guattari beschreiben es als eine Bewegung der Intensivierung: des Landens, des Eintauchens und Davonfliegens: »Atterrir, amerrir, s'envoler «. ${ }^{4}$ Der Begriff des Ritornells sucht auf die Frage zu antworten, wie durch die Wiederholung hindurch Veränderung geschehen, wie Ordnungen entstehen und Neues sich ereignen kann. Er ist deshalb, wie Deleuze unterstreicht, ein so wichtiger philosophischer Begriff.

1 Dieser Text erscheint in einer veränderten Fassung als ein Kapitel in: Astrid Deuber-Mankowsky, Queeres Post-Cinema. Yael Bartana. Su Friedrich. Todd Haynes. Sharon Hayes (Berlin: August, im Erscheinen).

2 L'abécédaire de Gilles Deleuze (R.: Pierre-André Boutang, Michel Parnat. FR 1996), 3, 28:10.

3 Ebd., 28:56.

4 Gilles Deleuze und Félix Guattari, Milles Plateaux (Paris: Minuit, 1980), S. 385. 
Seeing Red ${ }^{5}$ beginnt abrupt mit dem hohen $G$ eines Klaviers und der Aufnahme eines roten Tulpenbeets vor schwarzem Hintergrund. Das hohe $\mathrm{G}$ ist der erste Ton der Aria, welche die insgesamt 30 Variationen der Goldberg-Variationen von Johann Sebastian Bach einleitet. Es handelt sich um die rhythmische und schnell gespielte Aufnahme von Glenn Gould von 1981. Die Tonfolge wird mit einer präzise geschnittenen Bildermontage kombiniert, in jedem Bild taucht ein Rot auf. Es sind bewegte Bilder aus der Nachbarschaft in Brooklyn: ein rotes Graffiti, ein Junge mit einer roten Mütze, das roserne Halsband eines Hundes, eine Backsteinwand, der Schwanz einer Katze auf einem roten Kissen, ein Kran mit rotem Gitter, Kirschblüten, eine Orangenschale auf der Straße, die Neonwerbung eines Nagelstudios, ein leuchtendes Fahrrad, die roten Stiefel einer Frau, rosa Tulpen, Kinder im Park, dazwischen Aufnahmen von Su Friedrichs Torso in unterschiedlich roten T-Shirts, Blusen und Pullovern mit angestecktem Mikrophon bei sich zuhause. Erst nach drei Minuten wird der Titel eingespielt und man hört statt des Klaviers die dunkle Stimme von Su Friedrich, sieht sie in dem orangefarbenen T-Shirt in einem Ausschnitt vom Halsausschnitt bis zum angehefteten Mikrophon und hört, wie sie klagt: Sie wird fünfzig Jahre alt, steckt in einer Krise, hat ihre Gefühle nicht unter Kontrolle, nicht mehr als vor 30 Jahren, und erlebt sich zugleich als Kontrollfreak. Sie ärgert sich über ein Stück Papier auf dem Fußboden des Flurs, sie ärgert sich über ihre Mitbewohner_innen, die das Papier nicht wegräumen. Sie ärgert sich über sich selbst: Sie ist wütend und kann es nicht ändern. Nichts hat sich verändert. Dann setzt die Musik wieder ein.

\section{RINGELREIHEN}

In Tausend Plateaus führen Deleuze und Guattari das Ritornell über das Beispiel eines Kindes ein, das im Dunkeln Angst bekommt und sich beruhigt, indem es singt. Der Refrain ist der erste Ansatz von Ordnung im Chaos. Ein Zuhause entsteht, wenn ein Kreis um dieses Zentrum gebildet wird, als Beispiel nennen Deleuze und Guattari »das Ringelreihen der Kinder im Kreis herum ${ }^{6}{ }^{6}$ Dies könnte an den Zauberkreis erin-

\footnotetext{
5 Seeing Red (R.: Su Friedrich. USA 2005).

6 Gilles Deleuze und Félix Guattari, Tausend Plateaus. Kapitalismus und Schizophrenie (Berlin: Merve Verlag, 1992), S. 466.
} 
nern, den Johan Huizinga 1938 in seiner einflussreichen und für die folgenden Spieletheorien grundlegenden Studie Homo ludens. Vom Ursprung der Kultur im Spiel neben der Freiwilligkeit, der Differenz zum gewöhnlichen Leben und dem besonderen Spannungselement als viertes Element nannte, um das Spiel als Kulturform zu charakterisieren. ${ }^{7}$ Der Magic Circle bezieht sich für Huizinga auf die zeitliche und räumliche Abgegrenztheit des Spiels. Zwar ist auch für Huizinga das Spiel durch seine Wiederholbarkeit charakterisiert, doch setzt diese die Abgeschlossenheit eines gegen andere Tätigkeiten definierten Spiels voraus. Ein Spiel nimmt, wie Huizinga schreibt, »sogleich feste Gestalt als Kulturform an «. ${ }^{8}$ Für Huizinga »spielt sich ein Spiel $a b \ll ;^{9}$ es setzt eine gegebene Zeit voraus und ist nicht, wie das Ritornell, die Bewegung der Produktivität, die sich in der Wiederholung ereignet und durch die hindurch Zeit allererst entsteht. Tatsächlich geht es Deleuze und Guattari im Unterschied zu Huizinga und all den Spieletheorien, die sich auf Huizingas Studie beziehen, ${ }^{10}$ weder um das Spiel als eine Kulturform mit festen und verbindlichen Regeln, noch um die Entstehung der Kultur aus der Natur und auch nicht um die Abgrenzung von Spiel und Arbeit. Es geht ihnen vielmehr um die Entstehung von Zeit aus dem Chaos und um die Entstehung von Territorien und Gefügen (Agencements). So schafft man im Ringelreihe-Spiel nach Deleuze und Guattari keinen Zauberkreis im Sinne Huizingas, sondern »man kombiniert rhythmisierte Konsonanten und Vokale, die sowohl den inneren Kräften der Schöpfung wie den unterschiedlichen Teilen eines Organismus entsprechen $" .{ }^{11}$ Das Ringelreihen-Spiel erscheint hier nicht als eine feste Gestalt, sondern als Durchgang, als Passage. Der Kreis öffnet sich in eine Zukunft, man »bricht aus, wagt eine Improvisation «, man verbindet sich mit der Welt. »Am Leitfaden eines Liedchens geht man aus dem Haus. « ${ }^{12}$

7 Vgl. Johan Huizinga, Homo Ludens. Vom Ursprung der Kultur im Spiel (Reinbek bei Hamburg: Rowohlt, 2009), S. 18.

8 Ebd.

9 Ebd.

10 Siehe den Beitrag in diesem Band von Felix Raczkowksi, "Spielgrenzen und ihre Denkweisen«.

11 Deleuze u. Guattari, Tausend Plateaus, S. 424.

12 Ebd., S. 425. 
Su Friedrich drehte in den letzten siebenunddreißig Jahren über dreiundzwanzig Filme. Manche sind länger, 60 bis 81 Minuten, viele kürzer, zwischen 3 und 16 Minuten. Ihren ersten Film drehte sie 1978 auf Super-8, die folgenden Filme auf 16 mm, seit 2004 gibt es nur noch Videos. Friedrich ist dafür bekannt, dass sie in ihren Filmen Elemente des Dokumentarischen mit narrativen Elementen verbindet, mit Genres und Blickstrukturen spielt und Fragen der sexuellen Identität, ihre persönlichen Familien- und Krankheitsgeschichten in subtiler Weise mit Politischem verbindet und dafür immer neue formale Bewegtbildsprachen findet. Sie spielte eine zentrale Rolle in der Etablierung des avantgardistischen Queer Cinema und unterrichtet seit 1998 im Center for the Creative and Performing Arts an der Princeton University. Sie lebt fast ebenso lang in Brooklyn, das zum Schauplatz ihres letzten abendfüllenden Videos Gut Renovation (2012, 81 Min.) wurde und wo auch das kürzere Video Seeing Red (2005, 27 Min.) entstand. Seeing Red spielt mit dem Genre des Diary Films und macht dies auch explizit. In einer Zeit, in der, wie es 2006 in einer Rezension in der New York Times hieß, »man mit fast einer Million Videotagebücher, die auf Youtube.com gepostet sind, annehmen kann, dass die Menschen heute die Selbstaufnahme so natürlich finden wie Zähneputzen «. ${ }^{13}$ Seeing Red wird als Kommentar auf die sich ausbreitende Vlog-Kultur ausgelegt und zugleich als Fortsetzung von Friedrichs künstlerischer Auslotung des Zusammenspiels von Persönlichem, Politischem und des Mediums Film. Seeing Red markiert jedoch zugleich einen Bruch mit den früheren Filmen und stellt, wie ich im Folgenden vor dem Hintergrund von Deleuze und Guattaris Bezugnahmen auf das Spiel in der Genealogie von Gefügen und Walter Benjamins Verbindung von Spiel und zweiter Technik zeigen möchte, mehr dar als ein Spiel mit Genres: Friedrich betreibt darin das Filmen selbst als ein Spiel. Nicht als ein Spiel im Sinne von Huizinga, sondern mehr im Sinn des Ringelreihe-Spiels von Deleuze und Guattari: als eine Passage und eine Bewegung der Intensivierung, als ein Spiel mit Wiederholungen und ein Abschreiten von Variationen, als ein Spiel mit der Technik.

13 Stuart Klawans, "Midlife Fury, Glowing in Glorious Red «, The New York Times, 24 (September 2006): »With almost a million personal videos now posted on YouTube.com, we may guess that people today find self-recording to be as natural as tooth brushing $[\ldots]$.. 


\section{EIN GEFÜGE KONSTRUIEREN}

Begehren heißt, wie Deleuze und Guattari mit der Psychoanalyse und gegen die Psychoanalyse formulieren, ${ }^{14}$ ein Gefüge konstruieren. Mit der Psychoanalyse halten sie an dem realitätskonstituierenden Vermögen des Wunsches fest, gegen die Psychoanalyse wenden sie ein, dass man niemals ein abstraktes, also losgelöstes Objekt - eine Sache oder eine Person - begehrt, sondern immer ein konkretes Ensemble: Der Wunsch verläuft in einem konkreten Beziehungsgefüge und ist konstruktiv. Begehren heißt im wörtlichen Sinn agencer, zusammenfügen, konkrete Elemente (Farben, Gerüche, Landschaften, Dinge etc.) in ein Beziehungsgefüge bringen. Mit diesen Begriffen des Begehrens und des Gefüges (Agencement) unterminieren Deleuze und Guattari die abstrakte Gegenüberstellung von Subjekt und Objekt und damit auch jene von Arbeit und Spiel und von Technik und Natur. Sie knüpfen damit zugleich an die Versuche an, Technik nicht instrumentell und - damit verbunden - nicht anthropozentrisch zu denken, wie sie von Heidegger in seinem Aufsatz Die Frage nach der Technik und - was weniger bekannt ist von Walter Benjamin in seinen zerstreuten Ansätzen zu einer Philosophie der zweiten Technik in den 1930er Jahren vorgelegt worden sind. ${ }^{15}$ "Es ist ", so beschreiben Deleuze und Guattari das Verhältnis von technischen Elementen und gesellschaftlicher Maschine ganz analog zu dem Verhältnis von Begehren und Gefüge, anstelle von Begehren und Objekt, »das Prinzip jeder Technologie zu zeigen, dass ein technisches Element abstrakt und völlig unbestimmt bleibt, wenn man es nicht auf ein Gefüge bezieht, das es voraussetzt ".${ }^{16}$ Interessanterweise orientieren sich die beiden unterschiedlich geformten maschinellen Gefüge, die sich durch Tausend Plateaus ziehen, ihrerseits an unterschiedlichen Formen des Spiels: das Gefüge der Kriegsmaschine an dem alten chinesischen Strategiespiel Go, in dem es darum geht, einen offenen Raum einzuteilen und die Bewegung beständig zu halten, ohne Ziel, ohne Richtung, ohne Anfang und ohne Ende. Und das Gefüge des Staatsapparats am Schachspiel, in dem die Figuren codiert sind mit begrenzten Möglichkeiten der

14 Vgl. Monique David-Ménard, Deleuze und die Psychoanalyse. Ein Streit (Berlin und Zürich: Diaphanes, 2009).

15 Vgl. Astrid Deuber-Mankowsky, "Spiel und zweite Technik. Walter Benjamins Entwurf einer Medienanthropologie des Spiels", in Mediale Anthropologie, hg. v. Christiane Voss u. Lorenz Engell (München: Fink Verlag, 2015), S. 35-62. 
Bewegung und in dem es darum geht, einen begrenzten Raum einzuteilen. ${ }^{17}$

Su Friedrich fand ihre erste künstlerische Anerkennung 1981 mit Gently Down the Street, einem 14-minütigen Experimentalfilm ohne Ton. Aufflackernde weiße, in das Filmmaterial gekratzte Buchstaben und Wörter wechseln sich darin mit traumgleichen schwarzweißen Bildern von Frauen ab, die Frauen begehren, und erzeugen fragile Bilder lesbischen Begehrens. Die Zuschauerin taucht mit einer der weiblichen Figuren, die sich im Wasser eines Pools bewegt und dessen Umrandung gleichsam zum Bildrahmen wird, in diese Traumwelt ein und erwacht daraus erst wieder mit dem Ende des Films. Der nächste Film, mit dem sie bekannt wurde, war The Ties That Bind (1984, 55 Min.,16 mm) über die Geschichte von Su Friedrichs Mutter, die 1920 in Deutschland aufwuchs und, traumatisiert von den Erfahrungen des Krieges, 1947 mit ihrem US-amerikanischen Mann in die USA emigriert und dort, von ihm verlassen, drei Kinder alleine aufzieht. Friedrich ist in diesem Film die Gesprächspartnerin ihrer Mutter, erscheint aber ohne Stimme, allein über Sätze, die wieder in den Film gekratzt sind. Dann: Sink or Swim (1990, 48 Min., 16 mm), in dem die Beziehung zum abwesenden Vater und dessen Macht über die Tochter künstlerisch dokumentiert und rekonstruiert wird. Friedrich zeigt hier dem Alphabet rückwärts entlang Geschichten, die sie mit ihrem abwesenden Vater verbinden, die Geschichten sind aus der Sicht einer 3. Person erzählt, die Voice-over spricht eine Schauspielerin. Der nächste Film, Hide and Seek (1996, 63 Min., 16mm) unternimmt eine experimentelle Rekonstruktion von Erinnerungen an Kindheitserfahrungen von lesbischen Frauen in den 1960er Jahren in den USA und in Deutschland. Der Film folgt einem Drehbuch, das Friedrich zusammen mit Cathy Quinlan geschrieben hat. Ausschnitte aus Interviews folgen Szenen einer fiktiven, in die 1960er Jahre zurückversetzten Kindheit eines Mädchens, das sich in seiner Fremdheit in einer medialen durch und durch heteronormativen Umwelt den monkeys - den Affen - verwandt fühlt.

Erst in The Odds of Recovery (2002, 65 Min., 16 mm) erscheint Friedrich selbst im Bild. Der Film verknüpft Krankenbericht und Diary Film und zeichnet das genaue Portrait der Emotionen, die man im Pro-

\footnotetext{
17 Vgl. ebd., S. 484. 
zess nach einer Reihe von Operationen durchlebt: den Versuchen der Selbstbehandlung mithilfe von Tai Chi und gesundem Essen, Krisen in der Beziehung und in der Selbstwahrnehmung. Su Friedrich verwendet hier unter anderem Filmmaterial, das ihre Gespräche mit den Ärzt_innen im Krankenhaus zeigt, die sie mithilfe einer versteckten H8 Videokamera gefilmt und dann auf $16 \mathrm{~mm}$ übertragen hat. In diesem Film sieht und hört man die Regisseurin selbst über sich sprechen. Offensichtlich verstärkt die Technik des Videos die Tendenz der Regisseurin, den Platz hinter der Kamera durch jenen vor der Kamera zu ergänzen. Insofern könnte man den 2005 ganz auf Video gedrehten Film Seeing Red als vorläufigen Höhepunkt dieser Entwicklung betrachten. Die zentrale Differenz zu ihren früheren Filmen führt Su Friedrich selbst darauf zurück, dass sie mit einer neuen Technik - ganz mit Video - gearbeitet habe. Methodisch bestehe der größte Bruch darin, dass sie keinem Textbuch und keinem vorgängigen Plan mehr folge: Sie sei in ihr Studio gegangen, habe die Kamera aufgestellt und habe zu sprechen begonnen. Dies war möglich geworden, weil das Filmen mit Video zum einen billiger war und zum anderen der Schnitt am Computer einfacher. Nach der ersten Aufnahme gab es immer noch keinen Plan, aber eine einfache Regel: Die Regisseurin würde sich immer dann aufnehmen, wenn sie das Bedürfnis habe, etwas zu sagen und gleichzeitig würde sie in ihrer Umgebung Bilder sammeln, die etwas Rotes in sich haben. An einem Punkt sei dann die Entscheidung dazugekommen, Johann Sebastian Bachs GoldbergVariationen - Inkarnation und Höhepunkt barocker Variationskunst als Sound zu benutzen. Obwohl es technisch, wie sie unterstreicht, zwar leichter ist, am Computer zu schneiden, sei es dennoch ein langwieriger Prozess, den richtigen Rhythmus und narrativen Fluss zu finden. ${ }^{18}$ Und in der Tat stellt das Video eine komplexe Komposition dar, in der eine Farbe kontrapunktisch auf einen Ton antwortet, mehr ein Spielgefüge, das einem Abzählvers folgt, mehr ein obsessives Licht- und Tonspiel als eine Narration.

18 Katy Martin, "Su Friedrich Interviewed by Katy Martin« for Art World Magazine (Yishu Shijie), Shanghai, China and The Museum of Contemporary Art Shanghai (MOCA Shanghai), S. $7<$ http://www.katymartin.net/assets/su-friedrich-interview-by-katy-martin-sept08.pdf> [Zugriff 07.04.2015]. 
HIN - UND HERBEWEGEN, HÜPFEN, BESONDERS VOR FREUDE

Die Präzisierung, die Deleuze und Guattari von der Bewegungsqualität im Go-Spiel geben, in der ein offener Raum eingeteilt und in der die Bewegung selbst beständig wird, ohne Ziel, ohne Richtung, ohne Anfang und ohne Ende, knüpft, wie ein Blick in die Spieletheorien des 19. Jahrhunderts deutlich macht, an die etymologische Bedeutung des Wortes "Spiel« selbst an. Demnach bezeichnet »Spiel « nicht eine feste und abgegrenzte Kulturform, sondern eine sich wiederholende, rhythmische Bewegung, die zugleich Bewegen ist und Bewegt-Werden. »Es zeigt sich ", so heißt es in Buytendijks 1933 erschienenen Studie Wesen und Sinn des Spiels. Das Spielen des Menschen und der Tiere als Erscheinungsform der Lebenstriebe, »daß im Mittelniederländischen eine Bedeutung fortlebte, die auch im älteren Westgermanischen bestand, nämlich: sich in einer zuckenden Bewegung befinden, flimmern, hinund herbewegen, hüpfen, besonders vor Freude (lat. ensultare). « ${ }^{19}$

Buytendijk folgte damit einer in der Spieleliteratur gängigen Herleitung der Bedeutung des Spiels. So erinnerte der Völkerpsychologe und Sprachwissenschaftler Moritz Lazarus bereits 1883 in seinem grundlegenden Buch Über die Reize des Spiels daran, dass das Wort "spielen" auf das altdeutsche "spilan « zurückgeht, das mit dem Namen zugleich die Sache bezeichne: »eine leicht schwankende, ziellos schwebende Bewegung ${ }^{20}{ }^{20}$ Spielen ist darin vom menschlichen Spiel gelöst und wird als Manifestation des Lebens ausgelegt. »In diesem Sinne «, so kommentiert Lazarus weiter, »reden wir von einer Spielart, welche zwischen zweien Arten sich gleichsam in Bewegung befindet: von dem Herüberund Hinüberspielen des Weberschiffleins, von dem Spiel der Wasser im Springbrunnen und dem Spiel der Wellen in der Quelle, und von dem Spielraum, den eine Sache haben muß, um sich frei zu bewegen «. ${ }^{21}$ Lazarus hatte für seine Studie die von 1857-1875 in Deutschland veröffentlichte Literatur zum Spiel ausgewertet und mit der Anzahl der veröffentlichten Literatur im Bereich der Diätetik verglichen. Das Resultat: Die Spieleliteratur, die bis heute kaum erforscht wurde, übertraf mit Aus-

19 F.J.J. Buytendijk, Wesen und Sinn des Spiels. Das Spielen des Menschen und der Tiere als Erscheinungsform der Lebenstriebe (Berlin: Kurt Wolff Verlag, 1933), S. 18 .

20 Moritz Lazarus, Über die Reize des Spiels (Berlin: Dümmler Verlag, 1883), S. 19.

21 Ebd., S. 20. 
nahme der Zeitspanne von 1866-1870, in der, so Lazarus, die Impffrage heftig debattiert wurde, weit jene der Gesundheitsliteratur. ${ }^{22}$ Die Popularität des Spiels leitete sich, wie aus dem Zitat von Lazarus deutlich wird, aus der mediatisierenden Funktion her, die das Spiel für die Verschränkung von Lebendigem und Technischem einnahm. Bezeichnend dafür ist, dass das mechanische Hin und Her des Weberschiffchens in einem Zug mit dem Spiel der Wellen in der Quelle genannt wird. Während die Verbindung von Spiel und Sich-lebendig-Fühlen bereits im Zentrum des Kantischen Begriffs der ästhetischen Erfahrung und der Schiller'schen ästhetischen Erziehung des Menschen stand, so rückte das Spiel vor dem Hintergrund der Experimentalisierung des Lebens ${ }^{23}$ in der Mitte des 19. Jahrhunderts in die Nähe des Technischen: Die raum-zeitliche Struktur des Spielens im Sinne des »Spilans « konvergierte mit jener des Experiments: die "schwankende, ziellos schwebende Tätigkeit « wurde als ein »Immer-wieder-Tun « in die geregelte Wiederholung und das Durchgehen aller Variationen eines gleichen Vorgangs in einem definierten Setting eingebettet. So prägte Karl Groos, ein Schüler von Kuno Fischer, Philosoph und Psychologe den Begriff des »spielenden Experimentierens ", um das Spielen des Kleinkindes als ein Sich-in-der-Welt-Einrichten auszulegen. ${ }^{24}$ Die Nähe des so verstandenen Spiels zum Denken der Statistik und deren Anwendung in der Physiologie macht sich auch und zugleich in der von Johannes von Kries entwickelten "Spielraumtheorie « geltend. In dieser Theorie suchte der Physiologe Kries Kausalität und Wahrscheinlichkeit zu verbinden. ${ }^{25}$

Vermittelt über die Doppeldeutigkeit, die dem Spiel durch die Wiederentdeckung der etymologischen Bedeutung einer »leicht schwankenden, ziellos schwebenden Tätigkeit « oder, mit Buytendijk, eines »Flimmerns «, "Zuckens « und »Hin- und Herbewegens « zugesprochen wird, fungiert das Spiel, wie man zusammenfassend festhalten kann, seit dem 19. Jahrhundert als ein Medium zwischen Lebendigem und Technischem. Diese Ambiguität kulminiert in der Betonung des Rhythmus und

\footnotetext{
22 Vgl. ebd.

23 Siehe hierzu: Die Experimentalisierung des Lebens. Experimentalsysteme in den biologischen Wissenschaften 1850/1950, hg. v. Michael Hagner u. Hans-Jörg Rheinberger (Berlin: Akademie Verlag, 1993).

24 Karl Groos, Die Spiele der Menschen (Jena: Verlag Gustav Fischer, 1899), S. 7.

25 Johannes von Kries, Principien der Wahrscheinlichkeitsrechnung. Eine logische Untersuchung (Freiburg: J.C.B. Mohr, 1886).
} 
der Wiederholung als wesentliche Elemente des Spiels. Der Rhythmus verbindet das Moment des Mechanischen mit jenem des Lebendigen und wirkt als Scharnier zwischen Technischem und Lebendigem. Zugleich jedoch droht das Spiel im Mechanischen der Wiederholung die Anbindung an das Lebendige zu verlieren. Dies zeigt sich insbesondere in der Behandlung und Auslegung des Glücksspiels, das gleichermaßen mit Sucht, Geld, Automaten und Automatismus, Selbstzerstörung und Unproduktivität assoziiert wird. In eben dieser Ambiguität ging das Spiel 1920 als Ausdruck des Todestriebs und des Wiederholungszwangs in Freuds Jenseits des Lustprinzips ein.

Es sind nicht nur die Goldberg-Variationen, die in Seeing Red Wiederholungsschleifen hervorrufen. Es ist auch die Wiederholung des Settings: Su Friedrich, von der niemals das Gesicht zu sehen ist, in unterschiedlich roten Pullovern, Hemden, T-Shirts bei sich zuhause vor der Kamera sich beklagend, berichtend, auf und ab gehend, rauchend. Der Film ist als Diary, als Videotagebuch inszeniert. Es fehlen jedoch die Angaben von Daten und die Orientierung in der Zeit. Es gibt ein Heute, ein Gestern, ein »Als ich jung war», ein »Heute Abend « und »Jetzt werde ich gehen «, aber keine Entwicklung. "Words of wisdom don't come. Don't come «, ${ }^{26}$ wie es an einer Stelle heißt, es finden sich keine weisen Worte ein. Es ist nicht sicher, dass sich die gezeigten Szenen nacheinander abgespielt haben. Sie erscheinen aus dem Nichts, wie hin gewürfelt. Dieser Eindruck wird dadurch verstärkt, dass einzelne Bilder und Szenen mit ihren leuchtenden Rot-, Rosa- und Orangetönen wiederholt auftauchen, wie Teile eines Refrains - eines Ritornells. So erscheinen bereits im Vorspann alle acht Auftritte von Su Friedrich angedeutet durch acht Bildausschnitte aus diesen Auftritten, die verwoben sind mit Aufnahmen aus der Nachbarschaft in Brooklyn mit ihren Straßen und Parks, den Menschen, Tieren und Maschinen. Die visuelle Komposition nimmt die Form der Aria auf und spiegelt sie. Während Friedrich sich in ihren Wortbeiträgen nicht weiterbewegt, sich in ihrer Klage wiederholt, im Kreis zu drehen scheint, intensiviert sich das Spiel zwischen Tönen und bewegten Bildern in den Szenen, in denen nicht gesprochen wird und in denen Friedrich nicht zu sehen ist.

Besonders schön ist eine Szenenfolge, die auf einen Ausbruch von Ärger darüber folgt, dass dem Sollen kein Wollen, kein Können und

26 Seeing Red, 7:50. 
auch kein Tun folgt und das Sollen dennoch wie ein Imperativ bestehen bleibt, dem gegenüber man sich beständig fehlerhaft fühlt. ${ }^{27}$ Es folgt eine schnelle Folge von Pianotönen aus der 14. Variation der Goldberg-Variationen, die eingeleitet werden mit einem Bild, auf dem man im Vordergrund ein rotes Gitter und im Hintergrund fahrende Autos sieht. In der nächsten Einstellung bewegt sich die Kamera im schnellen Rhythmus der Musik an dem roten Gitter entlang, sodass die vorbeigleitenden roten Gitterstäbe mit den eingerahmten Bildausschnitten an einen bewegten Filmstreifen erinnern. Die Geschwindigkeit der Kamera steigert sich mit dem Rhythmus der Musik, man verliert die Kontrolle über das Bild, es geht über in Schwarz und endet mit dem Ausblick auf ein Stück blauen Himmel und dem Schwenk auf einen roten Kran. Nach einem kurzen Punkt der Ruhe wiederholt sich zunächst das Schwarz und wieder fährt die Kamera geschwind am Gitter vorbei, diesmal in die andere Richtung, die roten Stäbe leuchten auf und flitzen vorbei, seriell und sich wiederholend. Die Szene schließt auf der Ebene des Tons mit dem Ende der Variation und auf der visuellen Ebene mit einem Purzelbaum der Bilder eines roten sich bewegenden Stücks Plastik und schließlich in einem schwankenden Rot.

Über den Schnitt und die Komposition, das Zusammenspiel von Bild und Musik wird jene Bewegung aufgeführt, die Buytendijk, Lazarus und Groos mit der etymologischen Bedeutung des Spielens verbinden. Ein Flimmern, eine schwankende, ziellos schwebende Tätigkeit, eine Bewegung, die an Deleuze und Guattaris Beschreibung des Gefüges der Kriegsmaschine und des chinesischen Go erinnert, in der es darum geht, einen offenen Raum einzuteilen und eine Bewegung beständig zu halten.

\section{EIN IM MER-WIEDER-TUN}

In seiner Rezension von Karl Gröbers Kinderspielzeug aus alter Zeit. Eine Geschichte des Spielzengs entwarf Walter Benjamin 1928 die Grundzüge einer Theorie des Spiels. Er knüpfte dabei an den monumentalen Versuch von Karl Groos und dessen 546 Seiten umfassende Studie Spiele der Menschen aus dem Jahr 1899 an. Drei Aufgaben hätte sich eine solche Theorie nach Benjamin zu stellen: Erstens hätte sie eine

\footnotetext{
27 Ebd., 7:25.
} 
»Gestaltlehre der Spielgesten ${ }^{28}$ aufzustellen, zweitens der »rätselhaften Zweiheit Stock und Reifen, Kreisel und Peitsche, Murmel und Schieber, den Magnetismus, der sich zwischen beiden Teilen bildet, zu erforschen ${ }^{29}{ }^{29}$ Benjamin vermutet in diesen Spielen mit Unbelebtem ein Experimentieren mit ursprünglichen Rhythmen am Werk, in dem »wir zuerst unserer selbst habhaft werden ", und/oder ein Experimentieren bevor wir in der sexuellen Erfahrung des »Außerunsseins in der Liebe«, in das »Dasein und den oft feindlichen, nicht mehr durchdrungenen Rhythmus eines fremden, menschlichen Wesens « eingehen. ${ }^{30}$ Ausschlaggebend für unsere Frage und die Verbindung von Spiel und zweiter Technik, die er im Kunstwerkaufsatz vornehmen wird, ist jedoch die Schlussfolgerung, die er für die 3. Forderung zieht: »Endlich hätte eine solche Studie dem großen Gesetz nachzugehen, dass über allen einzelnen Regeln und Rhythmen die ganze Welt der Spiele regiert: Dem Gesetz der Wiederholung. « ${ }^{31}$

Mit Freud verknüpft Benjamin die Lust an der Wiederholung im Spiel des Kindes mit dem Trieb, der jenseits des Lustprinzips das Sexualleben der Erwachsenen bestimmt: "Wir wissen, dass sie dem Kind die Seele des Spiels ist, dass nichts es mehr beglückt als >noch einmal.»Wir wissen aber auch: »jedwede tiefste Erfahrung will unersättlich, will bis ans Ende aller Dinge Wiederholung und Wiederkehr, Wiederherstellung einer Ursituation, von der sie den Ausgang nahm «. ${ }^{32}$

Die Verschränkung von Glücksgefühl und Schrecken, die Benjamin in der Wiederholung ausmacht, findet er wieder im Doppelsinn des deutschen Wortes Spielen: »Dasselbe wiederholen wäre das eigentlich Gemeinsame. Nicht ein `So-tun-als ob‘, ein >Immer-wieder-tun`, Verwandlung der erschütterndsten Erfahrung in Gewohnheit, das ist das Wesen des Spielens. «33 Konsequent beschreibt Benjamin im Folgenden das Spiel als »Wehmutter « der Gewohnheit:

\footnotetext{
28 Walter Benjamin, "Spielzeug und Spielen. Randbemerkungen zu einem Monumentalwerk «, in ders., Gesammelte Schriften III, hg. v. Hella Tiedemann-Bartels (Frankfurt a.M.: Suhrkamp, 1991), S. 127-32 (S. 130).

29 Ebd.

30 Ebd., S. 131.

31 Ebd.

32 Ebd.

33 Ebd.
} 
Denn Spiel, sonst nichts ist die Wehmutter jeder Gewohnheit. Essen, schlafen, anziehen, waschen, müssen dem kleinen zuckenden Balg spielhaft, nach dem Rhythmus begleitender Verschen eingeimpft werden. Als Spiel tritt die Gewohnheit ins Leben, und in ihr, ihren starrsten Formen noch, überdauert ein Restchen Spiel bis ans Ende. Unkenntlich gewordene versteinerte Formen unseres ersten Glücks, unseres ersten Grauens, das sind die Gewohnheiten.

Und der Absatz endet fast wie ein Kommentar zu Friedrichs Seeing Red: »Und noch der trockenste Pedant spielt, ohne es zu wissen, kindisch nicht kindlich, am meisten, wo er am meisten Pedant ist. « ${ }^{34}$

In einer der Video-Diary-Szenen sieht man Su Friedrich bis zum Mund, in ihrem roten Pullover in einem weißen Fauteuil sitzend, das Frontispiz eines gebrauchten Gedichtbandes vor die Kamera haltend. Es zeigt das gezeichnete Portrait eines jungen Mannes mit rotem Schlapphut und hellem langen Haar und Bart, im Hintergrund eine grüne Wiese und ein blasser Himmel. Darauf steht in roten Buchstaben Walt Whitman und der Titel des Bandes Leaves of Grass. Friedrich liest zunächst den Vers, den sie mit zwanzig in den Band geschrieben hatte: »Nestled in the crook of my arm where the sweat crept on a summer day. « $^{35}$ Sie erinnert sich und lacht über die plötzliche Gegenwart der Vergangenheit. Sie schlägt ein Gedicht auf mit dem Titel »O me! O life! «. Walt Whitman gilt - trotz und wegen seines Patriotismus vielen als Prophet der Schwulenbewegung. Er verkörpert Intensität, Jugend, Romantik, lebendige Gegenwart, was die Fünfzigjährige ironisch kommentiert: »So much the sentiment of a twenty-year-old. « Und sie fügt an, sie könne sich vorstellen, dass man diese Gedichte von Walt Whitman mit zwanzig Jahren liest und liebt; wenn man zwanzig sei, fühle man sich »O me! O life!«. Und sie fährt ohne Pause fort: Sie scheine mit fünfzig dasselbe zu tun. Sie beginnt das Gedicht vorzulesen, während zugleich die Verse in roten Buchstaben und deren Schatten als Tiefendimension der Zeit auf dem Bild erscheinen und die schnellen Läufe des Pianos aus der Variation 29 zu hören sind:

O ich! O Leben! aus den wiederkehrenden Fragen danach,

34 Ebd.

35 Seeing Red, I 5:58. 
Nach den endlosen Zügen der Ungläubigen, nach Städten, mit Narren gefüllt, Nach mir selbst, der sich ewig selber tadelt, (denn wer ist närrischer als ich und wer ungläubiger? $)^{36}$

Su Friedrich liest die Antwort, mit der das Gedicht endet, nicht vor: »That the powerful play goes on, and you may contribute a verse! « ${ }^{37}$ Sie steigt stattdessen in das Spiel ein und kreiert ein eigenes Ritornell, »kindlich nicht kindisch .

\section{KLEINE VARIATIONEN}

Benjamin knüpfte in seinen Überlegungen zum Spiel an die Einübungstheorie von Karl Groos an, den seine Beobachtungen der kleinkindlichen Freude an rhythmischen Bewegungen, am Experimentieren mit sensorischen Reizen, an Abzählreimen, an Kinderliedern, am Tanzen etc. zu der These führte, dass Triebe, bei Menschen nicht weniger als bei Tieren, über spielerisches Experimentieren eingeübt werden. Groos geht in seiner Abhandlung über Die Spiele der Menschen von der spielenden Betätigung der sensorischen und motorischen Apparate und der spielenden Übung der seelischen Anlagen aus, über die das Kleinkind seiner eigenen Sinnesorgane habhaft wird, um dann auf die spielende Betätigung der Triebe zweiter Ordnung einzugehen. Triebe zweiter Ordnung sind für Groos jene Triebe, die für die Ausbildung des Verhaltens zu anderen Lebewesen zuständig sind: der Kampftrieb, die sexuellen Triebe und die Nachahmungstriebe. Dabei bezieht er sich bei der Bedeutung, die er der Nachahmung zukommen lässt, u.a. auf Gabriel Tarde und dessen 1890 erschienene Studie Les lois de l'imitation (1890), in der Tarde eine auf den Begriffen der Nachahmung und der Innovation beruhende evolutionäre Sozial- und Naturphilosophie präsentierte, auf die sich auch Deleuze an zentralen Stellen u.a. in Differenz und Wiederholung bezieht.

36 Walt Whitman, »O Ich! Oh Leben!«, in Grasblätter, hg. und übers. v. Jürgen Brôcan (München: Carl Hanser Verlag, 2009), S. 342-43 (S. 342). Wortlaut in Seeing Red: "O me! O life! Of the questions of these recurring, / Of the endless trains of the faithless, of cities fill'd with the foolish, / Of myself, forever reproaching myself, (for who more foolish than I, and who more faithless)? «.

37 Whitman, Grasblätter, S. 343: »Daß das kraftvolle Spiel weitergeht und du vielleicht eine Zeile beiträgst «. 
Und zwar dort, wo Deleuze seinerseits die Gewohnheit als Kontraktion und damit als erste Synthese der Zeit vorstellt. ${ }^{38}$

Tarde kommt nach Groos das Verdienst zu, gezeigt zu haben, dass der Begriff der Nachahmung dazu berufen sein könnte, »in der biologischen Psychologie eine ähnliche principielle Bedeutung zu gewinnen, wie der Begriff der Association in der älteren Seelenlehre «. ${ }^{39}$ Dabei fasse Tarde jedoch, wie Groos unterstreicht, die Nachahmung in einem weiteren Sinn auf, als es gewöhnlich geschehe: In diesem weiteren Sinn betrachte Tarde die Nachahmung "als Spezialfall des großen Weltgesetzes der Wiederholung (ondulation, génération, imitation sind die drei Formen der >repetition universelle $<) ~ «{ }^{40}$ Ganz ähnlich hebt Deleuze in seinem Kommentar zu Tarde hervor: »Die Nichtentsprechung von Differenz und Wiederholung begründet die Ordnung des Allgemeinen. In diesem Sinne legte Gabriel Tarde nahe, dass die Ähnlichkeit selbst nur eine verschobene Wiederholung sei: die wahre Wiederholung entspricht direkt einer Differenz gleichen Grades. ${ }^{41}$ Deleuze geht es hier - mit Tarde - um die kleinen Variationen, die in der Wiederholung summiert und integriert werden. ${ }^{42}$ Es geht um die Frage, wie eine immer genauere Entsprechung zwischen Differenz und Wiederholung hergestellt werden kann. Ganz freimütig formuliert Deleuze: »Die Gewohnheit entlockt der Wiederholung etwas Neues: die Differenz (die zunächst als Allgemeinheit gesetzt ist). « ${ }^{43}$ Dabei ist zu beachten, dass Deleuze, wenn er Gewohnheit mit Bergson als Kontraktion bestimmt, diese Kontraktion als qualitativen Eindruck auslegt. Erst als Empfundene kann die Kontraktion als Gewohnheit der Wiederholung etwas Neues - und das meint: eine Differenz - entlocken. Die lebendige Gegenwart, als welche Deleuze im Anschluss an Bergson und Kant die erste Synthese der Zeit bestimmt, ist mitsamt der sie begleitenden Lust das Neue, das die Gewohnheit als Verschmelzung von Wiederholungen im betrachtenden

\footnotetext{
38 Vgl. Gilles Deleuze, Differenz und Wiederholung (München: Wilhelm Fink Verlag, 1997), S. 99-110.

39 Groos, Spiele der Menschen, S. 361.

40 Ebd.

41 Deleuze, Differenz und Wiederholung, S. 44.

42 Vgl. ebd., Fußnote 4, S. 108.

43 Ebd., S. 103.
} 
Geist, das meint als passive »Form einer reinen Bestimmbarkeit (Raum und Zeit) « der Wiederholung entlockt. ${ }^{44}$

"Ich vermute, es wäre gut, wenn ich glauben könnte", so beginnt ein Filmtagebucheintrag auf dem das Rot dominiert, (man sieht nur die Einstellung auf den Bauch und den roten Pullover von Su Friedrich, das Spiel seiner Falten, wenn sie spricht und das in den Pullover geklemmte kleine schwarze Mikrophon),

dass ich eine bestimmte Anzahl von Gewohnheiten, Fähigkeiten und, ja, Werte und Interessen hätte und dass ich sie variieren könnte, so dass es nicht mehr darum ginge, dass man eine schlechte Person ist, die man in eine gute und damit in eine andere Person zu verändern versucht. Sondern zu denken, dass man, ja, dass man sehr enthusiastisch ist und das heißt, dass man manchmal manisch ist und exzessiv und ein anderes mal unglaublich fokussiert und aufmerksam - wie auch immer ... . ${ }^{45}$

Es wäre, so endet diese Rede, als ob diese Persönlichkeit und ihre verschiedenen Momente Variationen wären, die ein bestimmtes Interesse einen Refrain - zusammenhalten - in ähnlicher Weise, in der Bachs Goldberg-Variationen mit dem in der Aria eingeführten Motiv verfahren. Friedrich leitet hier eine präzise Reflexion auf das Zusammenspiel von Form und Inhalt ihres Filmes ein. Dies wird unterstrichen durch den Schnitt und die Komposition von Bild und Ton. So wird während ihrer Rede einmal der Blick auf den verblichenen roten Kamm einer Henne aus Gips gelenkt und ein anderes Mal das Bild auf den roten Schnabel einer Plastikente eingeblendet. Während die Rede in das Spiel der 9. Goldberg-Variation übergeht, zeigt die Kamera eine 50 Sekunden lange Nahaufnahme als eine Einstellung ohne Schnitt auf eine Wanderdrossel mit prächtig aufgeblähtem orangenen Bauch, die still und aufmerksam hinhört und sich hüpfend im Rhythmus bewegt. Anstelle ihres Gesangs hört man das Klavierspiel von Gould. ${ }^{46}$

Dieser präzise Umgang mit der Technik legt es nahe, über die Assoziation mit den kleinen Variationen von Tarde und Deleuze hinaus Benjamins Charakterisierung der zweiten Technik als »einmal ist keinmal «

\footnotetext{
44 Gilles Deleuze, Foucault (Frankfurt a.M.: Suhrkamp, 1987), S. 87.

45 Seeing Red, 14:10-14:50.

46 Ebd., 14:54-15:44. 
bis zur »unermüdlichen Variierung der Versuchsanordnung ${ }^{47}$ auf das spielerische und regelhafte Vorgehen von Friedrich in der Produktion und Postproduktion ihres Videos zu beziehen.

\section{ZWEITE TECHNIK UND KOSMOPOLITIK}

Groos Theorie des Spiels bezieht sich in weiten Teilen auf Schiller und dessen Einbindung des Spiels in eine idealistische Ästhetik. Dabei positionierte sich Groos zwar im Feld der " ̈̈sthetik von unten " ${ }^{48}$ das heißt, er interessierte sich für die entwicklungspsychologische Ausbildung der Wahrnehmung, hielt aber zugleich an den harmonischen Schönheitsidealen der idealistischen Ästhetik fest. Die zentrale Bedeutung, die der Frage des Ästhetischen, den Reizen, den Empfindungen und der Wahrnehmung in Groos' Konzept des experimentierenden Spielens zukam, war zweifellos ein wichtiger Grund für Benjamins Interesse an der Studie Die Spiele der Menschen. Im Unterschied zu Groos und auch zu Freud, von dem er das mechanische Moment in der Wiederholung und im Spiel übernahm, band Benjamin jedoch seine Theorie des Spiels in eine Theorie der zweiten Technik ein und führte Ästhetik, Spiel und Technik eng zusammen. So geht er von den Rhythmen aus, »in denen wir uns habhaft werden ${ }^{49}$ und nicht vom Kind und seinen Beziehungen zu den Objekten. Diese Rhythmen aber sind die gleichen Rhythmen wie jene, welche seit dem 19. Jahrhundert mit seinen technischen Innovationen der Dampfmaschine, der Elektrizität und den entsprechenden Aufzeichnungsmedien über das Spiel das Lebendige mit dem Technischen verbinden. Ähnlich wie später Simondon mit dem Begriff des Spielraums oder Deleuze und Guattari mit dem Begriff des Ritornells dachte auch Benjamin den Begriff der Technik zeitbasiert in Korrelation zu den Begriffen der Wiederholung und der Gewohnheit. Dabei assoziierte Ben-

47 Walter Benjamin, »Das Kunstwerk im Zeitalter seiner technischen Reproduzierbarkeit «, 2. Fassung, in ders., Gesammelte Schriften VII.1, hg. v. Rolf Tiedemann u. Hermann Schweppenhäuser (Frankfurt a.M.: Suhrkamp, 1991), S. 35084 (S. 359).

48 Siehe hierzu: Ästhetik von unten. Empirie und ästhetisches Wissen, hg. v. Marie Guthmüller u. Wolfgang Klein (Tübingen: Francke Verlag, 2006).

49 Vgl. oben, Fußnote 28. 
jamin die Gewohnheit mit Wohnen im Sinne der Frage eines in der Welt Eingerichtetseins und sich in der Welt Einrichtens. ${ }^{50}$

In seinen Texten aus der Mitte der dreißiger Jahre differenzierte Benjamin zwei technische Zeitalter, die zwei unterschiedliche Weisen des In-der-Welt-Eingerichtetseins und des Sich-in der-Welt-Einrichtens bezeichnen. Das eine nannte er »erste Technik«, das andere »zweite Technik «. ${ }^{51}$ In Anlehnung an den antiken Begriff der téchne bezeichnete Technik für Benjamin, ähnlich wie später für Heidegger, nicht allein die technischen Dinge, Maschinen und Apparate. Technik bedeutet für Benjamin jedoch auch mehr als das Können der Handwerker und der Künstler und das Verfahren und die Methode von zielgerichteten Tätigkeiten. Die Potenzialität der Technik liegt für Benjamin - und darin geht er über Heidegger hinaus - in ihrer Medialität. Er verbindet den Begriff der zweiten Technik mit der Frage der Wahrnehmungsästhetik als einer kollektiven Wahrnehmung. Insofern umfassen die Begriffe der ersten und zweiten Technik zugleich die zwei unterschiedlichen technischen Dispositive und die sich mit ihnen verändernde Weltaneignung. Die »zweite Technik « wird von Benjamin als eine »unermüdliche[...] Wiederholung der Versuchsanordnung " charakterisiert, ${ }^{52}$ ein Vorgehen, das er mit dem Begriff des experimentierenden Spielens assoziiert. Bezugspunkt für diese Engführung von Technik und Spiel bilden die historischen Veränderungen, die Benjamin in der Kunst und in den Wissenschaften beobachtet. $^{53}$

Für Benjamin zeichnet sich die Konstellation der zweiten Technik durch einen »ungeheuren Gewinn an Spielraum " aus. ${ }^{54}$ Ungeheuer ist dieser Spielraum ebenso wie die »ungeheure Entfaltung der Technik «5 in beiden Wortbedeutungen: ungeheuer groß sowie unheimlich, bedrohlich und ungewohnt.

Wenn Benjamin im Kunstwerkaufsatz notiert: »Der Ursprung der zweiten Technik ist da zu suchen, wo der Mensch zum ersten Mal und mit unbewusster List daran ging, Abstand von der Natur zu nehmen. Er

Vgl. Deuber-Mankowsky, Spiel und zweite Technik, S. 53-57.

Benjamin, »Das Kunstwerk «, S. 359.

Ebd.

53 Vgl. Walter Benjamin, "Erfahrung und Armut «, in ders., Gesammelte Schriften II.1, hg. v. Rolf Tiedemann u. Hermann Schweppenhäuser, S. 213-19 (S. 214).

54 Benjamin, »Das Kunstwerk «, S. 369.

55 Benjamin, »Erfahrung und Armut«, S. 214. 
liegt mit anderen Worten im Spiel «, ${ }^{56}$ so knüpft er dabei an seine früheren Ausführungen zum Verhältnis von Spiel und Gewohnheit an. Die zweite Technik verändert mit ihrem ungeheuren Gewinn an Spielraum alles. Sie eröffnet neue, unerhörte Intensitätsbewegungen und Möglichkeiten, aber auch eine ungeahnte Zerstörungskraft. Dies hat die »ungeheure Entfaltung der Technik « ${ }^{57}$ im ersten Weltkrieg in aller Deutlichkeit gezeigt. Für Benjamin kommt es nun darauf an, dass die Menschheit sich in dieser neuen technischen Umgebung im experimentierenden Spiel auf neue Weise habhaft wird. Vorgezeichnet sieht Benjamin dieses experimentierende Spielen nicht nur in den Verfahren der avantgardistischen Kunst, sondern auch in den neuen wissenschaftlichen Methoden der modernen Physik und der Soziologie, ihrer Interdisziplinarität und konstruktiven Verfahren und in der Bewegung des Bauhauses und des Neuen Bauens. ${ }^{58}$ Die Menschen müssen sich verjüngen und neu einrichten. Dabei gilt es an erster Stelle die Gewohnheit zu überwinden, die Technik als ein Instrument zu betrachten, das der Beherrschung der Natur diene. Diese anthropozentrische und teleologische Vorstellung bildet, wie Benjamin unterstreicht, ein zentrales Element des Dispositivs der ersten Technik und gehöre wie der wilhelminische Imperialismus und die Klassengesellschaft zu jenem Erbe, das eine wahrhaft kosmopolitische Gesellschaft überwinden müsse. Während, wie Benjamin schreibt, »das ein für allemal « für die erste Technik gilt, wo es um die »nie wiedergutzumachende Verfehlung und den stellvertretenden Opfertod « geht, gelte das »einmal ist keinmal für die zweite ", die es »mit dem Experiment und einer unermüdlichen Variierung der Versuchsanordnung zu tun « habe. ${ }^{59}$

"Menschen als Spezies stehen ", so formulierte Benjamin schon 1924 in der Einbahnstraße, »zwar seit Jahrtausenden am Ende ihrer Entwicklung; Menschheit als Spezies aber steht an deren Anfang. Ihr organisiert in der Technik sich eine Physis in welcher ihr Kontakt mit dem Kosmos sich neu anders bildet als in Völkern und Familien «. ${ }^{60}$ Dieser oft zitierte Satz erhält vor dem Hintergrund der Assoziation von

\footnotetext{
56 Benjamin, »Das Kunstwerk «, S. 359.

57 Benjamin, »Erfahrung und Armut «, S. 214.

58 Vgl. ebd., S. 215.

59 Benjamin, »Das Kunstwerk «, S. 359.

60 Walter Benjamin, »Einbahnstraße«, in ders., Gesammelte Schriften IV.1, hg. v. Tillman Rexroth (Frankfurt a.M.: Suhrkamp, 1991), S. 83-148 (S. 147).
} 
Spiel, Gewohnheit und Wohnen und dem Experimentieren mit ursprünglichen Rhythmen einen anderen Sinn: »Der Schauer echter kosmischer Erfahrungen " ist gebunden an Geschwindigkeiten, die - über die neuen Techniken - Fahrten ins »innere der Zeit «ermöglichen, wo man auf Rhythmen stößt, die ganz neue Möglichkeiten bereithalten, die mehr sind als die Summe von kleinen Variationen. ${ }^{61}$

Auch Su Friedrich bezieht sich in ihrem Film in einem ihrer Auftritte auf die Menschheit. Sie beklagt sich und klagt an. Sie sieht keine Möglichkeit, die Welt zu ändern und findet es zutiefst unfair. Von sechs Milliarden Menschen haben die meisten keine Arbeit, die sie ausreichend ernährt, sie sind von ihrer Arbeit gelangweilt oder ihre Arbeit macht sie unglücklich. Sie haben Schwierigkeiten in und mit ihren Familien. Friedrich schließt daraus, dass eine große Menge an Menschen auf diesem Planeten jeden Tag mehrere Male einen Gott anrufen, ihre Leben zu verbessern, oder Lotto spielen, Drogen nehmen, rauchen, ihre Liebsten betrügen... Man sieht sie von der Brust bis zum Kinn in einem roten Hemd, hinter ihr an der Wand hängen Stills aus Hide and Seek. Wieder werden Szenen aus der Nachbarschaft eingeblendet, diesmal sind es Leute, die in einem 99 Cent Store einkaufen. Su Friedrichs Stimme verschwindet und Glenn Goulds Interpretation der 22. Goldberg-Variation übernimmt die Tonspur. Die vom Speiseeis rot gefärbten Hände eines Eisverkäufers erscheinen im Bild, sie schaben im Rhythmus zur Musik aus einer großen Schale aus blinkendem Metall leuchtend rotes Eis und geben es kunstvoll in einen weißen kleinen Becher und nehmen einen Dollarschein entgegen. Die Kamera folgt den Händen und nimmt die schönen, fast tänzerischen Bewegungen auf, mit denen der Mann ein Bündel Dollarscheine ordnet, hin und her dreht und durchzählt. Es ist wieder eine lange Einstellung, sie dauert ohne Schnitt 84 Sekunden. ${ }^{62}$ Die Bewegung der rot gefärbten Hände wird schließlich abgelöst durch die Bewegungen eines kleinen Mädchens mit rotem Pulli und schwarzem Haar, das versucht, mit seinem Roller im Kreis zu fahren und einen gemeinsamen Rhythmus zu finden. Dies führt uns zur nächsten und letzten Frage: zu der Regel, nach der möglichst viel Rot im Bild erscheinen soll und zur Frage, welche Funktion diesem Rot im Lichte der hier vorgestellten Theorien des experimentierenden Spielens, der zweiten Tech-

\footnotetext{
61 Ebd.

62 Seing Red, 9:54-11:27. 
nik und des Ritornells zukommt. Wie verhält sich die Intensität der Farbe zur Feststellung, dass Veränderung mehr erfordert als die Summe aller kleinen Variationen?

\section{MASS UND RHYTHMUS: INTENSIVIERUNG}

Su Friedrich stellt diese Frage nach dem Rot in ihrem Video selbst, unmittelbar vor der Szene mit der das Video endet. Sie formuliert ihre Frage aus einem Nicht(mehr)wissen heraus. Eigentlich, so würde sie es ihren Studierenden beibringen, benutzt man kein Rot in Videofilmen und geht vorsichtig um mit Metaphern. Doch warum eigentlich kein Rot benutzen? »Blendendes, kitschiges, abscheuliches Rot, das über den Bildschirm blutet, das man nicht sehen will, und das keinerlei Information beinhaltet. « ${ }^{63}$ Sie öffnet dabei ihre Jacke und lässt das Rot ihres Pullovers über den Bildschirm fließen. Sie lässt die Frage offen, die Szene endet mit einer Ellipse: »Chances are when I...« - »Neue Möglichkeiten eröffnen sich, wenn ich....", es gibt keine andere Antwort.

Friedrich führt mit ihrer Frage ein selbstreferentielles Moment in den Film ein und weist auf die Ebene der Ästhetik hin. »Seeing Red « ist nicht nur eine Metapher, sondern eine Redewendung, ein Wortspiel. In einem Interview mit Katy Martin antwortet Friedrich auf die Frage, was »Seeing Red « bedeutet, dass es sich erstens auf den Affekt des Zorns und der Wut bezieht, zweitens sei es ihr darum gegangen, Farbe zu zeigen und dabei auf die Variationen der Farbtöne- und Schatten hinzuweisen, in denen eine Farbe erscheint. Und drittens stehe die Farbe Rot für Leidenschaft, für ein leidenschaftliches Verhältnis zu Welt im positiven Sinn des Wortes. ${ }^{64}$

Das Rot zeigt - mit Deleuze - eine Intensitätsdifferenz an. Die Farbe wird zum Ausdrucksmaterial, über die Farbe wird das Spiel von Su Friedrich expressiv. Wenn Deleuze bereits in Differenz und Wiederholung bei der Einführung der Gewohnheit als Kontraktion und erste Synthese der Zeit betonte, dass die Gewohnheit der Wiederholung nur unter der Bedingung etwas Neues - hier die lebendige Gegenwart - entlocken kann, dass sie als qualitativer Eindruck - und das meint als empfundene Differenz - ausgelegt wird, so erscheint dieses differentielle Spiel zwi-

\footnotetext{
63 Ebd., 23:21.

64 Martin, »Su Friedrich Interviewed by Katy Martin «, S. 10.
} 
schen quantitativen und qualitativen Größen in der Beschreibung des Ritornells wieder, wenn es in Tausend Plateaus heißt:

Ein Territorium gibt es, sobald es eine Expressivität des Rhythmus gibt. Ein Territorium wird durch das Auftauchen von Ausdrucksmaterien (Qualitäten) bestimmt. Nehmen wir zum Beispiel die Färbung von Vögeln und Fischen: Die Farbe ist ein Membranzustand, der von inneren Hormonbeständen abhängig ist: aber die Farbe bleibt funktional und transitorisch, solange sie mit einer bestimmten Art von Handlung verbunden ist (Sexualität, Aggression, Flucht). Sie wird dagegen expressiv, wenn sie eine zeitliche Konstanz und eine räumliche Reichweite bekommt, die sie zu einer territorialen oder vielmehr territorialisierenden Markierung machen - einer Signatur. ${ }^{65}$

Die zeitliche Konstanz setzt die Kontraktion der Zeit, oder eben die Gewohnheit und damit die Empfindung der Differenz in der Wiederholung voraus. Etwas Neues wird der Wiederholung durch eine Intensitätsdifferenz entlockt, die ebenso wie die Gewohnheit ein Quale und das meint, eine empfundene Differenz darstellt. Die Differenzierung zwischen funktionaler und expressiver Bedeutung der Farbe entspricht, wie wir nun festhalten können, der Differenz zwischen physikalischer Intensität, die gemessen werden kann und ästhetischer Bedeutung der Intensität, die sich als Quale der Messung entzieht: ${ }^{66}$ Während aus wissenschaftlicher Perspektive die Intensität als Variable einer Funktion im Bereich der quantitativen Größen verbleibt, stellt die Intensität im Bereich der Ästhetik, bzw. der Philosophie eine Qualität, oder, um einen anderen Begriff von Deleuze zu wählen, ein Empfindungswesen dar, ein "Être de sensations ", das meint, eine Empfindung, die dauert und zur Ausdrucksbewegung wird.

Diese Differenzierung erscheint wieder, wenn Deleuze darauf insistiert, dass der Rhythmus nicht dasselbe sei wie ein Maß. Dies wird bereits im ersten Satz der zitierten Passage deutlich, wenn es heißt: »Ein Territorium gibt es, sobald es eine Expressivität des Rhythmus gibt.« Deleuze und Guattari bekräftigen damit, was sie kurz zuvor in ihrem Text zum Verhältnis von Chaos und Rhythmus feststellten: »Die

Deleuze u. Guattari, Tausend Plateaus, S. 429.

Vgl. Astrid Deuber-Mankowsky, »Einleitung «, in Hermann Cohen, Das Prinzip der Infinitesimal-Methode und seine Geschichte. Ein Kapitel zur Grundlegung der Erkenntniskritik (Wien, Berlin: Turia+Kant, 2013), S. 7-70. 
Gemeinsamkeit von Chaos und Rhythmus ist der Zwischenraum... « und weiter:

Bekanntlich ist der Rhythmus weder Maß noch Kadenz, er ist nicht einmal eine unregelmäßige Kadenz: Nichts hat weniger Rhythmus als ein Militärmarsch [...]. Ein regelmäßiges oder unregelmäßiges Maß setzt eine codierte Form voraus, deren Maßeinheit wechseln kann, allerdings in einem nicht-kommunizierenden Milieu, während der Rhythmus das Ungleiche oder Inkommensurable ist, das ständig transcodiert wird. Das $\mathrm{Maß}$ ist dogmatisch, aber der Rhythmus ist kritisch, er verknüpft kritische Momente, oder er verknüpft sich mit dem Übergang von einem Milieu in ein anderes. ${ }^{67}$

Kommen wir zum Schluss zurück zum Ritornell und zum RingelreiheSpiel der Kinder. Ich habe zu Beginn dieses Beitrages darauf hingewiesen, dass das Ringelreihe-Spiel in der Auslegung von Deleuze und Guattari keinen Zauberkreis schafft im Sinne der Spieletheorie von Huizinga, sondern dass das Ringelreihe-Spiel im Sinne eines Ritornells eine Passage darstellt. Der Kreis, in dem rhythmisierte Konsonanten und Vokale kombiniert werden, wird im sprichwörtlichen Sinne geöffnet, man lässt jemanden eintreten oder tritt selbst aus dem Kreis heraus, "stürzt nach außen «. ${ }^{68}$ Dieses Nach-außen-stürzen kommt für Deleuze und Guattari mit der Neigung des Kreises überein, sich einer Zukunft zu öffnen. "Man bricht aus und wagt eine Improvisation «. ${ }^{69}$ Das Rot in Su Friedrichs Film deutet genau dies an: Das Rot ist das Expressiv-Werden des Rhythmus und insofern mehr als eine weitere Variation. Sie markiert mit dem Rot eine Grenze und schafft sich eine Bleibe. Deleuze und Guattari sehen in diesem Expressiv-Werden das Auftauchen der Kunst.

Anders als Deleuze interessierte sich Benjamin für die historische Veränderung der Wahrnehmung, die sich mit dem Übergang von der ersten zur zweiten Technik ereignete. Ein wichtiges Indiz für diese Veränderung war - parallel zu Film und Fotografie - das Aufkommen der Massen in den Großstädten. Massen aber waren für Benjamin, wie bereits Samuel Weber bemerkte, ${ }^{70}$ wesentlich Massenbewegungen. Samuel Weber deutet sie als Resultat, als logische Folgeerscheinung (corollary)

67 Deleuze u. Guattari, Tausend Plateaus, S. 427.

68 Ebd., S 425.

69 Ebd.

70 Vgl. Samuel Weber, Mass Mediauras: Form, Technics, Media (Stanford: Stanford University Press, 1996), S. 84. 
jener Bewegung der Auflösung, welche den Verfall der Aura und damit den Untergang der ersten Technik kennzeichnen. So verhalte sich die Aura zur Masse nicht nur als Einmaligkeit zu Vielheit, sondern - in räumlichen Begriffen - als fixierter Ort (Hier und Jetzt) zu einem Ort, der in eine unaufhörliche und komplexe Bewegung geraten ist. Tatsächlich verbindet Benjamin den Verfall der Aura im Kunstwerkaufsatz mit dem zunehmenden Wachstum der Massen in den großen Städten und der »zunehmenden Intensität ihrer Bewegungen «. ${ }^{71}$ Dass Benjamin an dieser Stelle den Ausdruck der Intensität benutzt, ist kein Zufall. Es zeigt vielmehr an, dass der Niedergang der Aura zugleich ein Übergang der Intensität vom Einmaligen und fixierten Hier und Jetzt - vom Punkt - in die unaufhörlichen Bewegungen der Massen ist. Dieser Übergang der Intensität vom Punkt auf die Bewegung markiert die historische Veränderung im Medium der Wahrnehmung. So ist das Medium, in dem sich die Wahrnehmung jener mit den Massen korrespondierenden Realität vollzieht, nicht mehr die Aura, sondern die Bewegungen der Massen. Entsprechend kommentiert Benjamin in der zweiten Fassung des Kunstwerkaufsatzes die »zunehmende Intensität « der Massenbewegungen mit dem Hinweis auf die veränderten Formen der Rezeption: »Die Dinge sich snäherzubringen` ist ein genauso leidenschaftliches Anliegen der gegenwärtigen Massen, wie es ihre Tendenz einer Überwindung des Einmaligen jeder Gegebenheit durch die Aufnahme von deren Reproduktion ist. $\ll^{72}$

In der letzten Szene ihres Films dreht sich Su Friedrich auf einem schwarzen Bürostuhl um sich selbst und zieht sich während der kreisenden Bewegung nach und nach eines ihrer orangenen, roten, rotkarierten etc. Hemden, Pullover und T-Shirts aus, die sie im Laufe des Films und der Videotagebucheinträge getragen hatte. $\mathrm{Zu}$ sehen ist wieder nur ihr Torso. Sie leitet das Spiel mit der Bemerkung ein, dass sie all das, was sie tut, im Licht dessen tut, dass sie eine Frau sei. Auf der Tonspur überla-

71 Benjamin, »Das Kunstwerk «, S. 355. In der »Kleinen Geschichte der Photographie«, aus der er die Passage über die Aura und deren Verfall wörtlich übernommen hat, steht dieser Satz noch nicht. Vgl. Walter Benjamin, »Eine kleine Geschichte der Photographie ", in Gesammelte Schriften II.1, hg. v. Rolf Tiedemann und Hermann Schweppenhäuser (Frankfurt a.M.: Suhrkamp, 1991), S. 368-85 (S. 378-79).

72 Benjamin, »Das Kunstwerk «, S. 355.

236 
gern sich, während Glenn Gould die 14. Variation spielt, all die Reden, die sie während ihrer Auftritte gesprochen hatte zu einem Turm aus Worten. Bis sie schließlich nur noch ein T-Shirt anhat. Sie gibt einen letzten Schwung und zieht sich auch das T-Shirt über den Kopf. Das Video endet mit dem Blick auf die Lehne des Stuhls, den nackten Rücken und die schwarzen Träger des BHs in einer leicht hin und her schwankenden Bewegung. Während das Bild schon nicht mehr zu sehen ist, sind während des Abspanns die letzten Läufe des Pianos noch zu hören. Und dann kann ein neues Spiel beginnen. 
Astrid Deuber-Mankowsky, »Variationen des Spiels: Seeing Red von Su Friedrich mit Deleuze, Guattari und Benjamin «, in Denkweisen des Spiels: Medienphilosophische Annäherungen, hg. v. Astrid Deuber-Mankowsky und Reinhold Görling, Cultural Inquiry, 10 (Wien: Turia + Kant, 2017), S. 213-37 <https://doi.org/10. 37050/ci-10_12>

\section{QUELLENANGABEN}

\section{BIBLIOGRAPHIE}

Benjamin, Walter, $\gg$ Einbahnstraße «, in ders., Gesammelte Schriften IV.1, hg. v. Tillman Rexroth (Frankfurt a.M.: Suhrkamp, 1991), S. 83-148

$\longrightarrow$ —Erfahrung und Armut «, in ders., Gesammelte Schriften II.1, hg. v. Rolf Tiedemann u. Hermann Schweppenhäuser, S. 213-19

— $\gg$ Eine kleine Geschichte der Photographie «, in Gesammelte Schriften II.1, hg. v. Rolf Tiedemann und Hermann Schweppenhäuser (Frankfurt a.M.: Suhrkamp, 1991), S. 368-85

— $\gg$ Das Kunstwerk im Zeitalter seiner technischen Reproduzierbarkeit «, 2. Fassung, in ders., Gesammelte Schriften VII.1, hg. v. Rolf Tiedemann u. Hermann Schweppenhäuser (Frankfurt a.M.: Suhrkamp, 1991), S. 350-84

— $\gg$ Spielzeug und Spielen. Randbemerkungen zu einem Monumentalwerk $\ll$, in ders., Gesammelte Schriften III, hg. v. Hella Tiedemann-Bartels (Frankfurt a.M.: Suhrkamp, 1991), S. 127-32

Buytendijk, Frederik Jacobus Johannes, Wesen und Sinn des Spiels. Das Spielen des Menschen und der Tiere als Erscheinungsform der Lebenstriebe (Berlin: Kurt Wolff Verlag, 1933)

David-Ménard, Monique, Deleuze und die Psychoanalyse. Ein Streit (Berlin und Zürich: Diaphanes, 2009)

Deleuze, Gilles, Differenz und Wiederholung (München: Wilhelm Fink Verlag, 1997)

Deleuze, Gilles und Félix Guattari, Milles Plateaux (Paris: Minuit, 1980)

_ Tausend Plateaus. Kapitalismus und Schizophrenie (Berlin: Merve Verlag, 1992)

Deuber-Mankowsky, Astrid, Queeres Post-Cinema. Yael Bartana. Su Friedrich. Todd Haynes. Sharon Hayes (Berlin: August, im Erscheinen)

— $\gg$ Einleitung $\ll$, in Hermann Cohen, Das Prinzip der Infinitesimal-Methode und seine Geschichte. Ein Kapitel zur Grundlegung der Erkenntniskritik (Wien, Berlin: Turia+Kant, 2013), S. 7-70

— $\gg$ Spiel und zweite Technik. Walter Benjamins Entwurf einer Medienanthropologie des Spiels «, in Mediale Anthropologie, hg. v. Christiane Voss u. Lorenz Engell (München: Fink Verlag, 2015)

Groos, Karl, Die Spiele der Menschen (Jena: Verlag Gustav Fischer, 1899)

Guthmüller, Marie u. Wolfgang Klein (Hg.), Ästhetik von unten. Empirie und ästhetisches Wissen (Tübingen: Francke Verlag, 2006) 
Hagner, Michael und Hans-Jörg Rheinberger (Hg.), Die Experimentalisierung des Lebens. Experimentalsysteme in den biologischen Wissenschaften 1850/1950 (Berlin: Akademie Verlag, 1993).

Huizinga, Johan, Homo Ludens. Vom Ursprung der Kultur im Spiel (Reinbek bei Hamburg: Rowohlt, 2009)

Klawans, Stuart, »Midlife Fury, Glowing in Glorious Red «, The New York Times, 24 (September 2006)

von Kries, Johannes, Principien der Wahrscheinlichkeitsrechnung. Eine logische Untersuchung (Freiburg: J.C.B. Mohr, 1886)

Lazarus, Moritz, Über die Reize des Spiels (Berlin: Dümmler Verlag, 1883)

Martin, Katy, »Su Friedrich Interviewed by Katy Martin« for Art World Magazine (Yishu Shijie), Shanghai, China and The Museum of Contemporary Art Shanghai (MOCA Shanghai), S. 7. <http://www.katymartin.net/assets/su-friedrich-interview-by-katy-martinsept08.pdf> [Zugriff 07.04.2015]

Weber, Samuel, Mass Mediauras: Form, Technics, Media (Stanford: Stanford University Press, 1996)

Whitman, Walt, »O Ich! Oh Leben!«, in Grasblätter, hg. und übers. v. Jürgen Brôcan (München: Carl Hanser Verlag, 2009), S. 342-43

\section{FILMOGRAPHIE}

L'abécédaire de Gilles Deleuze (R.: Pierre-André Boutang, Michel Parnat. FR 1996)

Seeing Red (R.: Su Friedrich. USA 2005) 